\title{
Effects of displacement and distortion of potential surfaces on absorption and emission spectra of MPV oligomer in solutions
}

\author{
C.H. Chang ${ }^{\text {a,e, * }, ~ J . C . ~ C h a n g ~}{ }^{\mathrm{a}, \mathrm{b}}$, R. Chang ${ }^{\mathrm{a}}$, J.H. Hsu ${ }^{\mathrm{a}, \mathrm{b}}$, W.S. Fann ${ }^{\mathrm{a}, \mathrm{b}}$, M. Hayashi ${ }^{\text {a }}$, J. Yu ${ }^{\mathrm{c}}$, \\ J.C. Jiang ${ }^{\text {a }}$, S.H. Lin ${ }^{\text {a }}$, Y.Z. Lee ${ }^{\mathrm{d}}$, K.R. Chuang ${ }^{\mathrm{d}}$, S.A. Chen ${ }^{\mathrm{d}}$ \\ ${ }^{a}$ Institute of Atomic and Molecular Sciences, Academia Sinica PO Box 23-166, Taipei, 106 China \\ ${ }^{\mathrm{b}}$ Department of Physics, National Taiwan University Taipei, China \\ ${ }^{\mathrm{c}}$ Department of Chemistry, National Kaoshiung Normal University, Kaoshiung, China \\ ${ }^{\mathrm{d}}$ Department of Chemical Engineering, National Tsing-Hwa University, Hsinchu, China \\ ${ }^{\mathrm{e}}$ Department of Chemistry, National Taiwan University, Taipei, China
}

Received 17 August 1998; received in revised form 11 December 1998; accepted 14 December 1998

\begin{abstract}
We report here the vibrational modes for MPV oligomer in solvents of toluene as well as chloroform, obtained by studying the photo-luminescence and absorption spectra. In analyzing the absorption and emission spectra, it is found that both displacements and distortions of vibrational modes are important in fitting the observed spectra. (C) 1999 Elsevier Science S.A. All rights reserved.
\end{abstract}

Keywords: Displacement; Distortion; MPV oligomer

\section{Introduction}

In the recent years, Poly ( $p$-phenylene vinylene) (PPV) and its derivatives have attracted much interests due to the progress in their synthesis [1,2] and their potential applications in electro-optic devices [3,4]. Several studies have focused on understanding the electronic and morphological structures of these polymers. Recently, Yu. et. al. [5] have applied the molecular orbital theory as well as molecular exciton theory to calculate the singlet-to-singlet and triplet-to-triplet electronic transitions in PPV-oligomer with various chain lengths. These calculations reasonably explained the experimental observations [5]. Principally, a similar methodology has been applied to obtain the absorption spectra of PPV and dimethoxy-PPV oligmers by Cornil et. al [6]. In addition, Yu et. al. [7] and Cornil et. al. [8] have also studied the vibronic structure and obtained the corresponding absorptions spectra. Notably, in most situations, the samples under study are in the form of thin film. It is therefore reasonable to assume that chain lengths are widely distributed instead of being uniform. Moreover, the interaction between the neighboring chains is also expected to lead to changes in the spectra. Yu et. al. have therefore studied the effect of nonuniform distribution of chain lengths [9]. The absorption and photoluminescence excitation (PLE) spectra, thus obtained, could describe the experimental result adequately. While the interchain effect was studied with a kinetics model which offers explanation of the temperature effect in both the absorption and PL spectra; however, in order to understand the details of interactions, it is necessary to separately study the intrachain and interchain effects. For this purpose, we have measured the absorption (Abs), PL spectra and radiative life time for an oligomer, MPV in this paper. Due to its simplicity in electronic states, detail of the vibrational contribution to the spectra can be revealed. In our theoretical analysis, we will emphasize the importance of the distortion of potential surface that can lead to the asymmetric profiles of emission and absorption spectra.

\footnotetext{
* Corresponding author. E-mail: richang@po.iams.sinica.edu.tw
} 


\section{Experimental}

The experiments were performed for MPV solutions in toluene as well as in chloroform with concentration $2.5 \times 10^{-6}$ M. Fig. 1 shows the structure of MPV. The absorption spectrum is taken from a spectrophotometer Hitachi model U-3200, while PL spectrum from HITACHI model F-4010. Details of the sample preparation and measurement of the spectra have been reported previously [9-12]. The results are shown in Fig. 2a,b,c together with theoretical calculation that will be explained in Section 3. Notice the emission spectra are displayed as $I(\omega) / \omega^{3}$ and absorption spectra as $\alpha(\omega) / \omega$ where $\alpha(\omega)$ is the absorption coefficient. Both solutions have asymmetric profiles of absorption and PL. The width of absorption is slightly greater than that of PL. In particular, the PL spectrum in toluene exhibits more the vibronic feature than that in chloroform.

It is well known that for displaced potential surfaces, the absorption and PL spectra will exhibits the mirror image relation. As can be seen from the experimental absorption and PL spectra, this relation is not observed in our spectra. In this paper, we shall consider the effect of the distortion of potential surfaces on absorption and PL spectra.

In addition, the time-correlated single-photon counting technique is employed to measure the fluorescence decay time. The radiative decay time $\tau_{\text {rad }}$ is then deduced from the measurement of quantum efficiency. For MPV in toluene and chloroform, $\tau_{\text {rad }}=1.3 \mathrm{~ns}$ and $1.45 \mathrm{~ns}$, respectively.

\section{Theoretical analysis and discussions}

Here we will present briefly the basic theoretical approach used in the analysis of the spectra. In general, the absorption coefficient for the electronic transition $a \rightarrow b$ can be expressed as

$$
\alpha_{a b}(\omega)=\frac{4 \pi^{2} \omega}{3 a c \hbar}\left|\vec{\mu}_{b a}\right|^{2} \sum_{\nu} \sum_{\nu^{\prime \prime}} P_{a \nu}\left|\left\langle\Theta_{b \nu^{\prime \prime}} \mid \Theta_{a \nu}\right\rangle\right|^{2} D\left(\omega_{b \nu^{\prime \prime} a \nu}-\omega\right)
$$

in which the adiabatic approximation and Condon approximation have been used. In Eq. (1), $\vec{\mu}_{b a}$ denotes the electronic transition moment, $P_{a \nu}$ the Boltzmann factor, $\left|\left\langle\Theta_{b \nu}{ }^{\prime \prime} \mid \Theta_{a \nu}\right\rangle\right|^{2}$ the Frank-Condon factor, $a$ the factor which describes the medium effect, $\left(\nu, \nu^{\prime \prime}\right)$ the vibrational quantum numbers, $c$ the speed of light and $D\left(\omega_{b \nu^{\prime \prime} a \nu}-\omega\right)$ the lineshape function. Assuming the wave function $\Theta$ to be that of harmonic oscillator and expressed as a product of wave functions of each mode

$$
\Theta_{a \nu}=\prod_{j} \chi_{a \nu_{j}}\left(Q_{j}\right) ; \Theta_{b \nu^{\prime \prime}}=\prod_{j} \chi b \nu_{j}^{\prime \prime}\left(Q_{j}^{\prime \prime}\right)
$$

with an inhomogeneity distribution of electronic energy gap

$$
g\left(\omega_{b a}\right)=\sqrt{1 / 2 \pi d^{2}} \exp \left(-\left(\omega_{b a}-\bar{\omega}_{b a}\right)^{2} / 2 d^{2}\right)
$$

one obtains [13]

$$
\alpha_{a b}(\omega)=\frac{2 \pi \omega}{3 a c \hbar}\left|\bar{\mu}_{b a}\right|^{2} \int_{-\infty}^{\infty} \mathrm{d} t \exp \left[\frac{i t}{\hbar}\left(E_{b}-E_{a}-\hbar \omega\right)-\mathrm{d}^{2} t^{2} / 2\right] \prod_{j} G_{j}(t)
$$

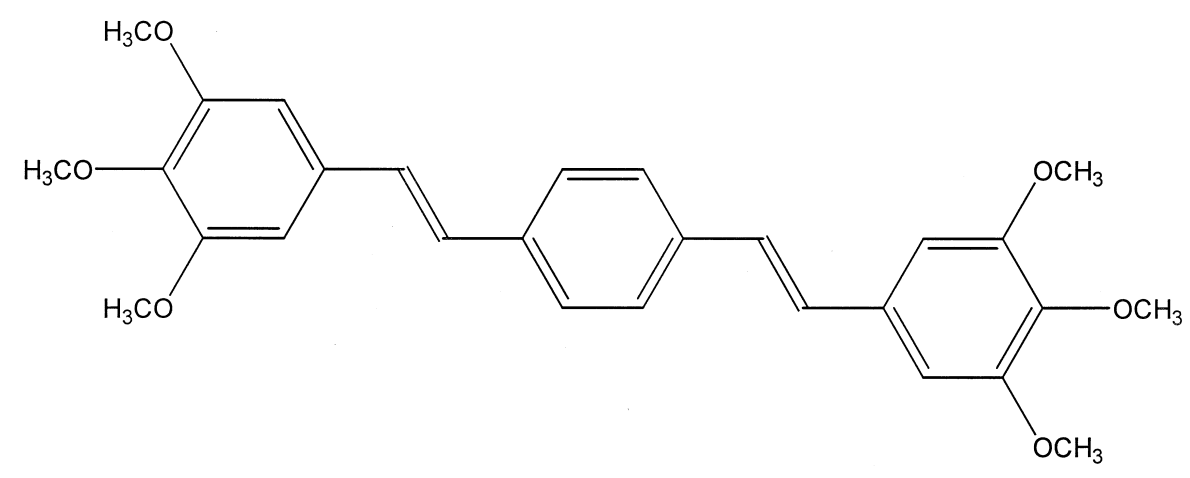

Fig. 1. The molecular structure of MPV oligomer. 
where

$$
G_{j}(t)=\sum_{\nu_{j}} \sum_{\nu_{j}^{\prime \prime}} P_{a \nu_{j}}\left|\left\langle\chi_{b \nu_{j}^{\prime \prime}} \mid \chi_{a \nu_{j}}\right\rangle\right|^{2} \times \exp \left[i t\left\{\left(\nu_{j}^{\prime \prime}+\frac{1}{2}\right) \omega_{j}^{\prime \prime}-\left(\nu_{j}+\frac{1}{2}\right) \omega_{j}\right\}\right] .
$$

and $\omega_{j}$ and $\omega_{j}^{\prime \prime}$ are the oscillator frequencies of the $j$ th mode in the electronic states $a$ and $b$, respectively. In a more explicit form, Eq. (5) can take the form

$$
G_{j}(t)=\frac{2 \beta_{j} \beta_{j}^{\prime \prime} \sinh \left(\hbar \omega_{j} / 2 k T\right)}{\sinh \left(\lambda_{j}\right) \sinh \left(\mu_{j}^{\prime \prime}\right)} \times \frac{\exp \left[-\beta_{j}^{2} \beta_{j}^{\prime \prime 2} \Delta{Q_{j}^{\prime \prime}}^{2} /\left(\beta_{j}^{\prime \prime 2} \operatorname{coth}\left(\lambda_{j} / 2\right)+\beta_{j}^{2} \operatorname{coth}\left(\mu_{j}^{\prime \prime} / 2\right)\right)\right]}{\left[\left(\beta_{j}^{\prime 2} \operatorname{coth}\left(\mu_{j}^{\prime \prime} / 2\right)+\beta_{j}^{2} \operatorname{coth}\left(\lambda_{j} / 2\right)\right)\left(\beta_{j}^{\prime 2} \tanh \left(\mu_{j}^{\prime \prime} / 2\right)+\beta_{j}^{2} \tanh \left(\lambda_{j} / 2\right)\right)\right]^{1 / 2}}
$$

where

$$
\beta_{j}=\left(\frac{\omega_{j}}{\hbar}\right)^{1 / 2}, \Delta Q_{j}^{\prime \prime}=Q_{j}^{\prime \prime}-Q_{j}, \lambda_{j}=i t \omega_{j}+\frac{\hbar \omega_{j}}{2 k T}, \mu_{j}^{\prime \prime}=-i t \omega_{j}^{\prime \prime},
$$

and $\Delta Q_{j}^{\prime \prime}$ denotes the normal coordinate displacement. For emission spectra, one can obtain a similar expression given as

$$
I_{b a}(\omega)=\frac{2 \pi a \omega^{3}}{3 c \hbar}\left|\vec{\mu}_{b a}\right|^{2} \int_{-\infty}^{\infty} \mathrm{d} t \exp \left[\frac{i t}{\hbar}\left(E_{b}-E_{a}-\hbar \omega\right)-d^{2} t^{2} / 2\right] \prod_{j} \bar{G}_{j}(t)
$$

where $\bar{G}_{j}(t)=G_{j}^{*}(t)$ with $\omega_{j}$ and $\omega_{j}^{\prime \prime}$ exchanged.

For the case of pure displacement, i.e., $\beta_{j}=\beta_{j}^{\prime \prime}$ and $\Delta Q_{j}^{\prime \prime} \neq 0, G_{j}(t)$ reduces to

$$
G_{j}(t)=\exp \left[\sum_{j=1}^{N} S_{j}\left\{\left(\bar{n}_{j}+1\right)\left(e^{-i t \omega_{j}}-1\right)+\bar{n}_{j}\left(e^{i t \omega_{j}}-1\right)\right\}\right] .
$$

In this case, we obtain the well known relations

$$
\alpha_{a b}(\omega)=\frac{2 \pi \omega}{3 a c \hbar}\left|\vec{\mu}_{b a}\right|^{2} \int_{-\infty}^{\infty} \mathrm{d} t \exp \left[\frac{i t}{\hbar}\left(E_{b}-E_{a}-\hbar \omega\right)-\mathrm{d}^{2} t^{2} / 2+\sum_{j=1}^{N} S_{j}\left\{\left(\bar{n}_{j}+1\right)\left(e^{-i t \omega_{j}}-1\right)+\bar{n}_{j}\left(e^{i t \omega_{j}}-1\right)\right\}\right],
$$

and

$$
\begin{aligned}
I_{b a}(\omega)= & \frac{2 \pi a \omega^{3}}{3 c \hbar}\left|\vec{\mu}_{b a}\right|^{2} \int_{-\infty}^{\infty} \mathrm{d} t \exp \left\{\frac{i t}{\hbar}\left(E_{b}-E_{a}-\hbar \omega\right)-\mathrm{d}^{2} t^{2} / 2\right\} \\
& \times \exp \left[\sum_{j=1}^{N} S_{j}\left\{\left(\bar{n}_{j}+1\right)\left(e^{i t \omega_{j}}-1\right)+\bar{n}_{j}\left(e^{-i t \omega_{j}}-1\right)\right\}\right],
\end{aligned}
$$

where the Huang-Rhys factor $S_{j}=\omega_{j} \Delta Q^{\prime 2} / 2 \hbar$ and $\bar{n}_{j}=1 /\left(\exp \left(\hbar \omega_{j} / 2 k T\right)-1\right)$. It is noteworthy that the emission spectrum $I_{b a}(\omega) / \omega^{3}$ and absorption spectrum $\alpha_{a b}(\omega) / \omega$ in the pure displacement case have a mirror image relation. We will thus include the distortion effect if the asymmetry is observed.

In choosing the optically active mode in simulation, we take second derivatives for absorption as well as PL spectra so that the primary vibronic frequency can be estimated. We found the values are about $1400 \mathrm{~cm}^{-1}$. Previous IR and Raman spectra also justify the existence of vibrational modes around this range [14-16]. Additionally, we also consider modes around $800 \mathrm{~cm}^{-1}$ and $200 \mathrm{~cm}^{-1}$ respectively. The mode $800 \mathrm{~cm}^{-1}$ is determined not just by fitting the spectra. Previous investigations have been done for benzene, styrene and stilbene [17-20] whose structures are closely related to that of MPV molecule and several Raman modes near $800 \mathrm{~cm}^{-1}$ were found in these compounds. The choice of low frequency mode $200 \mathrm{~cm}^{-1}$ is essential in explaining the large Stokes shift observed in the spectra. The choice of vibrational modes is also consistent with our previous molecular orbital calculations [5,7]. We fix the electronic energy at $E_{b}-E_{a}=24725 \mathrm{~cm}^{-1}$ in this calculation.

We first apply Eqs. (10) and (11) to calculate the spectra for MPV oligomer in toluene. These equations consider only the displacements without any distortions. We choose three modes $\omega=1400,800$, and $187 \mathrm{~cm}^{-1}$, and Huang-Rhys factors $S=1.2,0.38$ and 3.41 , respectively. The chosen value of $d$ of inhomogeneity is $225 \mathrm{~cm}^{-1}$. The results shown in Fig. 2a demonstrate that PL and absorption spectra cannot be fitted simultaneously. We ascribe the discrepancy to the potential surface distortion and apply Eqs. (6) and (8) to simulate its effect. We assume that the ground (excited) state potential 
surface consists of three optically active modes with frequencies $\omega=1400(1480), 800(800)$, and 187(205) $\mathrm{cm}^{-1}$, coupling constants are $S=1.2,0.38$ and 3.41, respectively and $d=225 \mathrm{~cm}^{-1}$. Here, in the expression $S_{j}=\omega_{j} \Delta Q^{\prime 2} / 2 \hbar, \omega_{j}$ is the ground state vibrational frequency. The result thus calculated and shown in Fig. $2 \mathrm{~b}$ can fit the experiment quite well.

In a similar way, we calculate the spectra for MPV in chloroform. The modes we choose are $\omega=1350(1420), 825(825)$, and $200(200) \mathrm{cm}^{-1}$, the corresponding coupling constants are $1.32,0.37$ and 4.41 , respectively and $d=225 \mathrm{~cm}^{-1}$. The result is shown in Fig. 2c. It has to be remarked that the absolute value of the low frequency mode is not so sensitive but has to be chosen so that $S \omega$ which controls the magnitude of Stokes shift is with a fixed value.

(a)

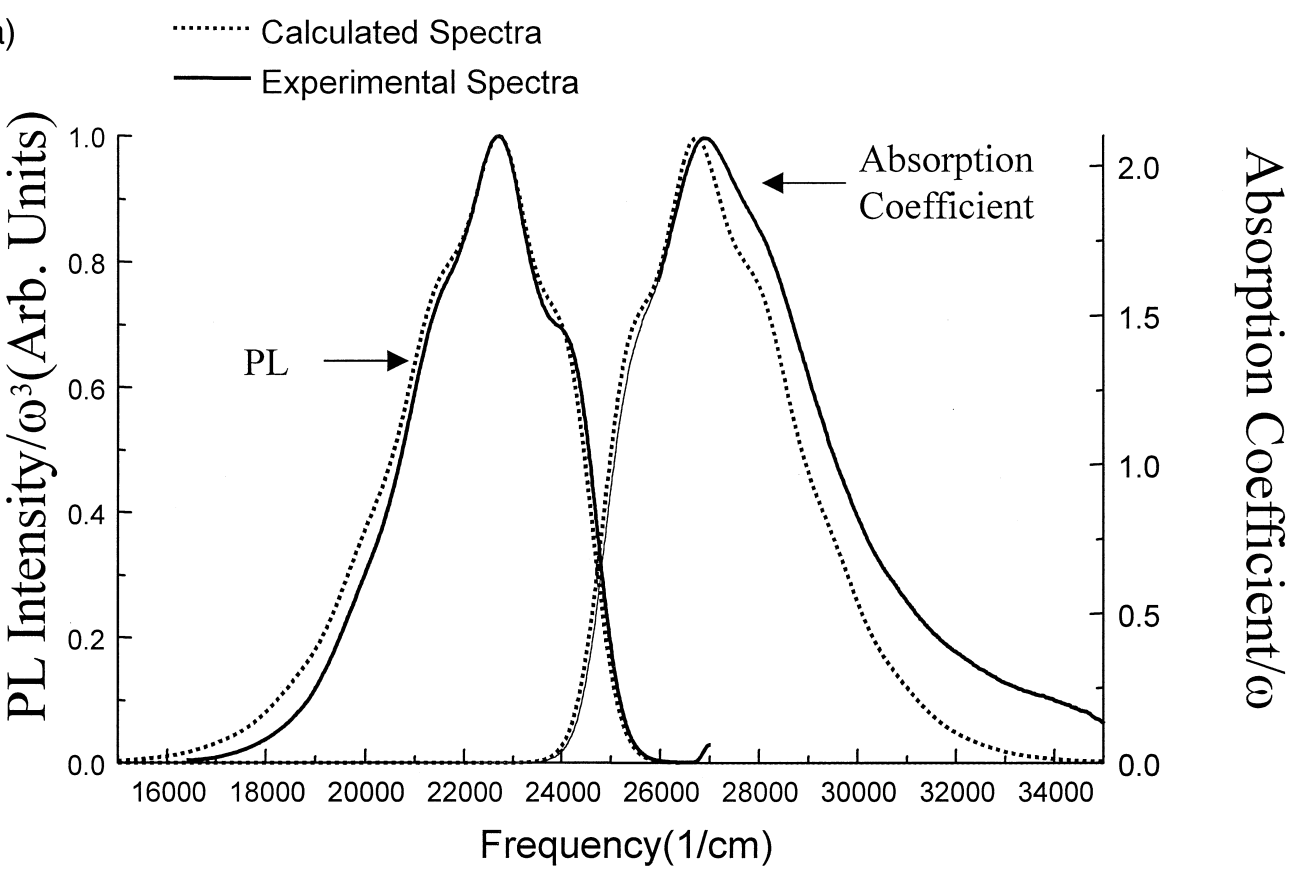

(b)

Calculated Spectra

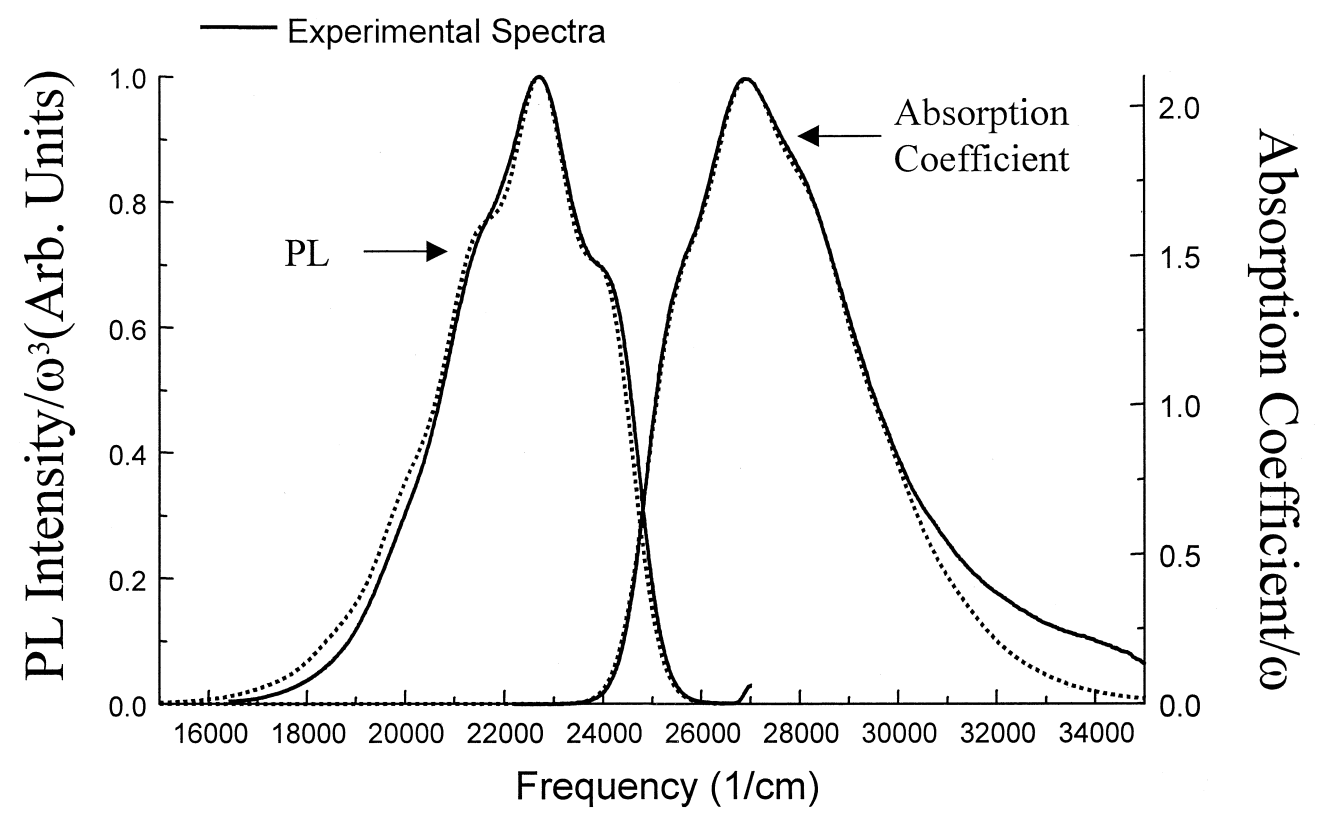

Fig. 2. (a) The experimental and calculated (without distortion) photoluminescence and absorption spectra of MPV in solvent toluene. (b) The experimental and calculated (with distortion) photoluminescence and absorption spectra of MPV in solvent toluene. (c) The experimental and calculated (with distortion) photoluminescence and absorption spectra of MPV in solvent chloroform. Experimental and calculated spectra are denoted by solid and dashed lines, respectively. 


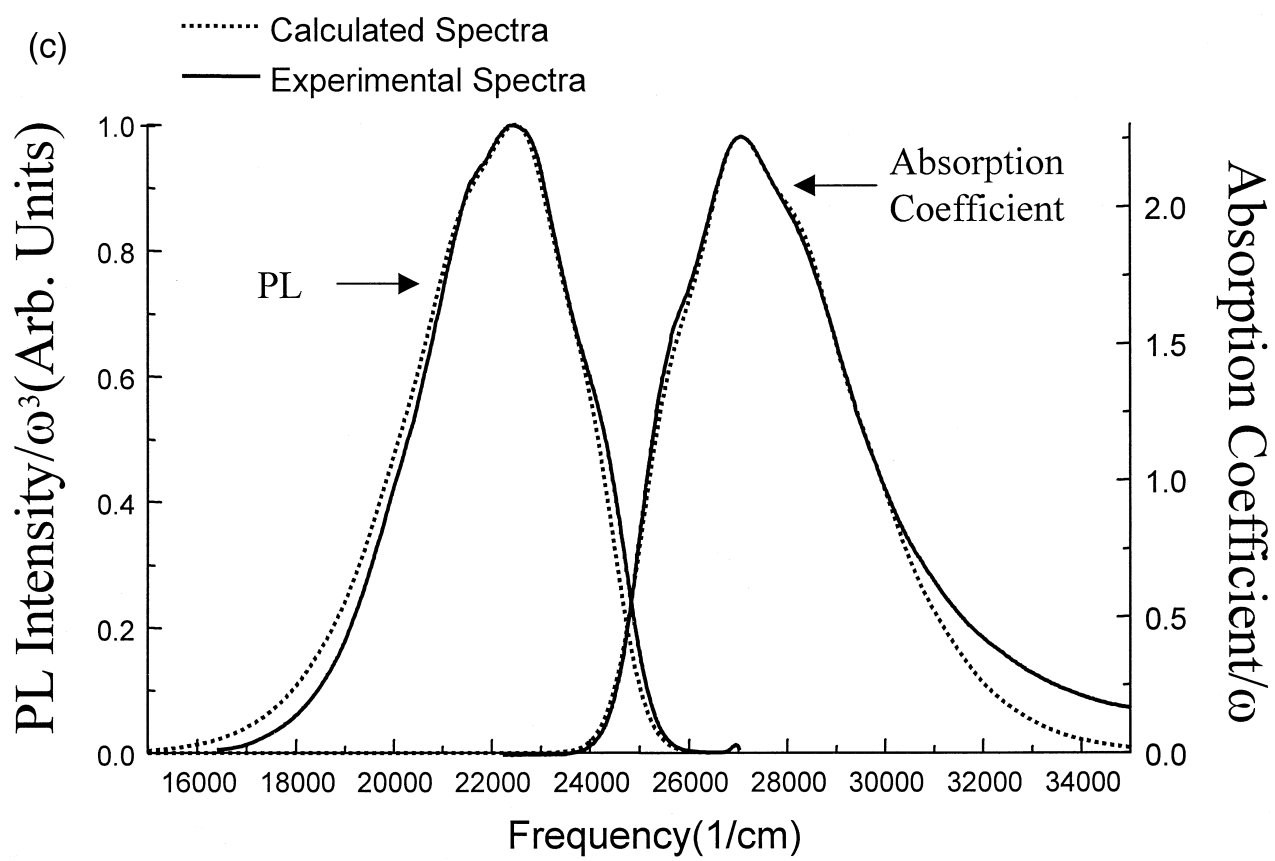

Fig. 2 (continued).

Another mechanism has been suggested to interpret this asymmetry effect. It is that there are more than one excited electronic states, and the higher excited states contribute less to luminescence due to the thermal distribution. However, for this small oligomer, we can imagine that the higher electronic state should be largely separated from the first one. Previous molecular orbital calculation reported by Yu et al. [5] has justified this fact. Therefore, the small asymmetry observed in the spectra is probably not due to multiple electronic states. However, an alternative possibility is that the conformation of excited state is different from that of ground state, i.e., the isomer transformation. This will also lead to two or more closely separated states and thus the absorption spectrum will be broader than the PL spectrum.

In order to consider the possibility of conformation change in the excited state, we calculate the radiative life time $\tau_{\text {rad }}$ of MPV in both solvents by applying the Strickler-Berg formula [21] that is correct only for one excited electronic state. The formula reads

$$
\frac{1}{\tau_{\text {rad }}}=2.880 \times 10^{-9} n^{2}\left\langle\tilde{\omega}^{-3}\right\rangle_{A V}^{-1} \int \alpha_{a b}(\omega) \frac{\mathrm{d} \omega}{\omega}
$$

where

$$
\left\langle\tilde{\omega}^{-3}\right\rangle_{A V}^{-1}=\frac{\int I_{b a}(\omega) \mathrm{d} \omega}{\int \omega^{-3} I_{b a}(\omega) \mathrm{d} \omega},
$$

and $n$ is the refractive index of the solvents. The calculated lifetimes for MPV in toluene and chloroform are 1.45 and 1.5 ns, respectively, which agree with the experimental results adequately. This result indicates that the PL spectra observed are definitely from the first excited state and the asymmetry is most likely due to the distortion of vibration modes.

\section{Conclusions}

We have determined the vibrational modes for the MPV oligomer in solutions. It is found that the potential surface is displaced and distorted when the oligomer is excited.

\section{Acknowledgements}

This work is supported in part by the National Science Council of ROC and in part by Academia Sinica. 


\section{References}

[1] T. Granier, E.L. Thomas, D.R. Gagnon, F.E. Karasz, R.W. Lenz, J. Polym. Sci. B 24 (1986) 2793.

[2] D.D.C. Bradley, R.H. Friend, H. Linderberger, S. Roth, Polymer 27 (1986) 1709.

[3] R.H. Friend, D.D.C. Bradley, P.D. Townsend, J. Phys. D 20 (1987) 1367.

[4] I.D.W. Samual, B. Crystall, G. Rumbles, P.L. Burn, A.B. Holmes, R.H. Friend, Chem. Phys. Lett. 213 (1993) 472.

[5] J. Yu, W.S. Fann, F.J. Kao, D.Y. Yang, S.H. Lin, Synth. Met. 66 (1994) 143.

[6] J. Cornil, D. Beljonne, R.H. Friend, J.L. Bredas, Chem. Phys. Lett. 223 (1994) 82.

[7] J. Yu, J.H. Hsu, K.R. Chuang, C.L. Chao, S.A. Chen, F.J. Kao, W.S. Fann, S.H. Lin, Synth. Met. 74 (7) (1995) .

[8] J. Cornil, D. Beljonne, Z. Shuai, T.W. Hagler, I. Campbell, D.D.C. Bradley, J.L. Bredas, C.W. Spangler, K. Mullen, Chem. Phys. Lett. 247 (425) (1995) .

[9] J. Yu, M. Hayashi, S.H. Lin, K.-K. Liang, J.H. Hsu, W.S. Fann, C.I. Chao, K.R. Chuang, S.A. Chen, Synth. Met. 82 (1996) 159.

[10] T.W. Campbell, R.N. McDonald, J. Org. Chem. 24 (1246) (1959) .

[11] Z. Yang, I. Sokolik, F.E. Karasz, Macromolecules 26 (1188) (1993) .

[12] S.C. Moratti, R. Cervini, A.B. Holmes, D.R. Baigent, R.N. Friend, N.C. Greenham, J. Gruner, P.J. Hamer, Synth. Met. 71 (2117) (1995) .

[13] S.H. Lin, J. Chem. Phys. 44 (3759) (1966) .

[14] B. Tian, G. Zerbi, K. Muellen, J. Chem. Phys. 95 (1991) 3198.

[15] B. Tian, G. Zerbi, K. Muellen, J. Chem. Phys. 95 (1991) 3191.

[16] G. Zerbi, E. Galbiati, M.C. Gallazzi, C. Castiglioni, M. Del Zoppo, R. Schenk, K. Mullen, J. Chem. Phys. 105 (1996) 2509.

[17] G. Varsányi, SzÕke, Vibrational Spectra of Benzene Derivatives, Academic Press, New York, 1969.

[18] D.A. Condirston, J.D. Laposa, J. Mol. Spectrosc. 63 (466) (1976) .

[19] A. Marchand, J. Quintard, Spectrochim. Acta 36 (941) (1978).

[20] C.H. Choi, M. Kertesz, J. Phys. Chem. A. 101 (1997) 3823.

[21] S.J. Strickler, R.A. Berg, J. Chem. Phys. 37 (1962) 814. 